\title{
Application of lower fluence rate for less microvasculature damage and greater cell-killing during photodynamic therapy
}

Published online: 25 March 2005

(c) Springer-Verlag London Limited 2005

\begin{abstract}
During the process of photodynamic therapy (PDT), problems arise such as stasis or occlusion of microvasculature, tumor oxygen depletion, and photosensitizer bleaching. This study shows that the first problem could be reduced by using a lower fluence rate light source in PDT. Microvasculature damage was studied experimentally in hematoporphyrin derivativemediated PDT against light fluence rate, and, to some extent, less microvasculature damage was induced under $75 \mathrm{~mW} / \mathrm{cm}^{2}$ illumination than under $150 \mathrm{~mW} / \mathrm{cm}^{2}$. Histology of vessels at the end of PDT showed that vessel damage could be observed in both groups, indicating that the microvasculature would eventually be damaged as long as the administration of light fluence was sufficient and regardless of the illuminating fluence rates. Thus microvasculature damage induced by low fluence rate illumination could also be effective in tumor control after PDT. The cell-killing experiment was performed in vitro and designed so that cell-killing rate was influenced only by light characteristics. The higher cellkilling rate caused by $75 \mathrm{~mW} / \mathrm{cm}^{2}$ illumination indicated that lower fluence rate light could enhance the light absorbency or decrease the bleaching of photosensitizer.
\end{abstract}

Keywords Cell fraction surviving - Light fluence · Microvasculature damage - Photodynamic therapy · $\mathrm{RBC}$ column diameter

The online version of the original article can be found at http:// dx.doi.org/10.1007/s10103-004-0310-1

T. $\mathrm{Xu}(\square) \cdot \mathrm{X} . \mathrm{Wu}$

School of Precision Instruments and Opto-Engineering,

Tianjin University, 235\#, Tianjin, 300072, China

E-mail: xutao780606@hotmail.com

Tel.: + 86-22-27409460

Fax: + 86-22-83520973

Y. $\mathrm{Li}$

Laser Laboratory of Medical, Tianjin Medical University, Tianjin, 300070, China
Abbreviations BSA: Bovine serum albumin - HpD:

Hematoporphyrin derivative - OD: Optical

density - PDT: Photodynamic therapy $\cdot \mathrm{PO}_{2}$ : Partial

pressure of oxygen - RBCCD: RBC column

diameter $\cdot$ ROS: Radical oxygen species

\section{Lasers Med Sci (2004) 19:150-154}

The content of page 154 did not appear in print. The complete article is reprinted here.

\section{Introduction}

Photodynamic therapy (PDT) has been shown to be a very effective treatment for cutaneous tumors, esophageal tumors, and some other cancers, and is believed to act through cytotoxic singlet oxygen (a radical oxygen species [ROS]). Singlet oxygen is formed when a photosensitizer is excited by light and transfers its energy to the molecular oxygen in tissues. The oxidization of the singlet oxygen may be the principal way to destroy the tumor cells in PDT. Photosensitizer, tissue oxygen, and irradiation light are considered to be the three elementary factors of PDT $[1,2]$.

Excitation of the photosensitizer with high-intensity laser pulses, which are of high fluence rate, can cause a transient change of light absorption [3]. The light dose absorbed in PDT will depend on several effects, such as saturation of the ground-state absorption, excitation to upper excited singlet and triplet states, and permanent photobleaching of the sensitizer. Hence, it is presumed that there are some internal relations between the photobleaching and the light fluence rate.

The status of tumor oxygen in PDT is determined by blood flow rate associated with the oxygen supply rate, the oxygen consumption of cellular metabolism, the photochemical consumption of oxygen, and the status of vessel occlusion or blood stasis [4]. An 
experimental result showed that six out of eight carcinomas had a sharp decrease in median partial pressure of oxygen $\left(\mathrm{PO}_{2}\right)$ when the light source in PDT was $150 \mathrm{~mW} / \mathrm{cm}^{2}$, while only three out of nine carcinomas had a slight decrease under the same dose treatment of $30 \mathrm{~mW} / \mathrm{cm}^{2}$ light [5]. There was a significant decrease of the median of $\mathrm{PO}_{2}$ values (from $25.4 \mathrm{mmHg}$ before PDT to $9.2 \mathrm{mmHg}$ after) and a significant increase of the percentage of values less than $2.5 \mathrm{mmHg}$ after $150 \mathrm{~mW} / \mathrm{cm}^{2}$ PDT (from $6.7 \%$ before PDT to $35.1 \%$ after), while the values varied from 19.5 to $37.4 \mathrm{mmHg}$ and the percentage values from $10 \%$ to $8.1 \%$ after $30 \mathrm{~mW} / \mathrm{cm}^{2}$ PDT. All these facts indicated that oxygen in tissues could be easily depleted at high fluence rates, whereas it could be preserved at low fluence rates. Similar conclusions have been made by theoretically employing a tissue oxygen consumption and diffusion model, and in general the higher the fluence rate, the greater the decrease in the oxygen concentration $[6,7]$. It is assumed that oxygen can be preserved at low fluence rates, as there is less damage to the microvasculature [8]. This needs to be verified further.

Some researchers predicted that low fluence rates cause more tumor cure than high fluence rates with the same total fluence [9-11]. Cell-killing models of PDT related to the light dose have been introduced to show the relationship between the fluence rate and the $50 \%$ killing dose of PDT [12]. Some experiments concerning the microscopic cell-killing rate at different fluence rates are suggested for further research.

\section{Materials and methods}

Animal models

Wistar rats weighing $200 \pm 10 \mathrm{~g}$ (Experimental Animal Center of PLA General Hospital, Beijing, China) were obtained at least 1 week before experimentation, and were then housed in a room with subdued lighting and fed with a standard pellet diet and water, with project approval from the Animal Experiment Center of Tianjin Medical University. A chondrosarcoma tumor cell line was transplanted into the right flank of each rat. The tumors that grew to be approximately 6-7 $\mathrm{mm}$ in size within about 5 days after transplantation were used in this experiment.

\section{Suspension of mammary cancer cell}

Mammary cancer cell MDAMB 543 was obtained from the tumor laboratory of PLA General Hospital and was cultured in vitro in $90 \%$ RPMI 1640 incubation medium (GibcoBRL, USA), 10\% bovine serum albumin (BSA), and $1 \%$ penicillin and streptomycin at $37^{\circ} \mathrm{C}$. Then the cancer cells were incubated in $5 \% \mathrm{CO}_{2}$.
Microvasculature damage in PDT against light fluence rate

The rats were given hematoporphyrin derivative $(\mathrm{HpD})$ via tail vein injection at a dose of $2.5 \mathrm{mg} / \mathrm{kg}$ and were anesthetized with $50 \mathrm{mg} / \mathrm{kg}$ sodium pentobarbital $24 \mathrm{~h}$ later. Shaved and depilated, their right legs were fixed (as described in [13]), to prevent them from moving during the light treatment. With a frequency-doubling semiconductor laser (Photoelectricity Engineering Department, Tsinghua University, Beijing, China) acting as light source, the output light was coupled in an optical silex fiber with a core diameter of $200 \mu \mathrm{m}$ and then delivered to illuminate the treatment field containing tumors at a continuous wave model. The light was $532 \mathrm{~nm}$ in wavelength and could be set at two different fluence rates, 75 and $150 \mathrm{~mW} / \mathrm{cm}^{2}$, monitored by a LLM-3 power meter (National Institute of Metrology, Beijing, China). The rats were divided into two group$\mathrm{s}$ - the $75 \mathrm{~mW} / \mathrm{cm}^{2}$ illuminated group and the $150 \mathrm{~mW} /$ $\mathrm{cm}^{2}$ group - and placed on their back on a temperaturecontrolled pad heated by a far-infrared heater. As depicted previously [14], the tumor microvasculature was observed and studied. Having been slit and flooded with saline, the right flank muscle was covered with a clear plastic film to prevent drying. An inverted microscope (IX70; Olympus, Japan) incorporated with a digital camera (D-360L; Olympus, Japan) was used to visualize the tumor microvasculature, and the images were recorded for later analysis. The distribution of the vasculature was observed and little fatty regions with an arteriole and venule in pairs were chosen for the study, based on the RBC column diameter (RBCCD). RBCCD was measured through the playback of the recorded images every $4 \mathrm{~min}$ for $32 \mathrm{~min}$ for the $75 \mathrm{~mW} / \mathrm{cm}^{2}$ illuminated group, and every $2 \mathrm{~min}$ for $16 \mathrm{~min}$ for the $150 \mathrm{~mW} / \mathrm{cm}^{2}$ illuminated group. Relative RBCCD at time $t$ was recorded, as defined by the equation:

$\mathrm{RBCCD}_{\text {rel }}=\frac{\operatorname{RBCCD}(t)}{\operatorname{RBCCD}(0)}$

where $\operatorname{RBCCD}(t)$ and $\operatorname{RBCCD}(0)$ are the absolute column diameter (a mean of 5-7 points) at time $t$ and just before PDT, respectively. Ten rats were used in each different experimental group and the mean relative RBCCD at time $t$ was thought to reflect the status of the microvasculature after some dose illumination. The RBCCD is a measurement of the inner lumen of the vessel, and reductions in RBCCD without a change in wall diameter indicated that aggregates had formed at the inner vessel wall [14].

Three tumor samples from both experimental groups were collected immediately after the completion of illumination for histological study, rinsed with warm saline and placed in $95 \%$ alcohol at $4{ }^{\circ} \mathrm{C}$ for $72 \mathrm{~h}$ before they were dehydrated and embedded in paraffin wax. A section cutter was used to slice the samples into sections at $0.004 \mathrm{~mm}$, and then the sections were dewaxed, 
rehydrated, stained, and mounted for observation and photography by a biological microscope (BX50; Olympus, Japan).

Cell-killing in PDT against light fluence rate

The cultured MDAMB 543 cells were made into singlecell suspensions at a concentration of $1.0 \times 10^{5} / \mathrm{ml}$ with $0.25 \%$ trypsin (Sigma, St Louis, MO, USA). Three 96well culture plates (Costar, Cambridge, MA, USA) were used to inoculate the suspension with $0.2 \mathrm{ml}$ per well and placed in the $5 \% \mathrm{CO}_{2}$ incubator at $37^{\circ} \mathrm{C}$ for $16 \mathrm{~h}$, after which the supernatant culture media was removed.Then $\mathrm{HpD}$ as the photosensitizer, diluted in RPMI 1640 incubation medium to a concentration of $0.004 \mathrm{mg} / \mathrm{ml}$, was added to the wells. The culture plates were then placed in the $5 \% \mathrm{CO}_{2}$ incubator at $37^{\circ} \mathrm{C}$ and completely protected from light. About $4 \mathrm{~h}$ later, two culture plates were illuminated by $150 \mathrm{~mW} / \mathrm{cm}^{2}$ laser for $30 \mathrm{~s}$ and $75 \mathrm{~mW} / \mathrm{cm}^{2}$ laser for $60 \mathrm{~s}$, respectively, while the third plate was still kept protected from light as a control group. After illumination the culture plates were replaced in the incubator for $24 \mathrm{~h}$, and then $0.1 \mathrm{mg}$ diphenylterrazolium bromide (Sigma, St Louis, MO, USA) was added to the wells before another 4-h culture. After this culture, supernatant fluid having been removed and $0.15 \mathrm{ml}$ dimethylsufoxide solution $(1 \mathrm{mg} / \mathrm{ml})$ having been added, culture plates were then shaken slightly for $10 \mathrm{~min}$ and the optical density (OD) of all the wells was measured by an enzyme-labelling device (318-MC; Xinke, Shanghai, China). The fraction of cells surviving was defined as the ratio of the mean OD of experimental groups to that of the control group.

\section{Statistics}

To study the microvasculature damage against light fluence rate, the relative RBCCD values were compared between the two experimental groups after illumination with the same light dose, using the Wilcoxon signed rank test at $95 \%$ confidence to show the different effects on microvasculature damage at the two fluence rates. For the cell-killing rate against light fluence rate, the same statistical method was used.

\section{Result}

Changes of RBCCD in PDT at different fluence rates

The RBCCD changes of venules and arterioles both occurred under the illumination of 75 and $150 \mathrm{~mW}$ laser light. Data collected from the image playback of experimental groups are summarised in Fig. 1. In Fig. 1a, there was an indication that under low fluence rate illumination there occurred less microvasculature damage than under high fluence rate illumination in the
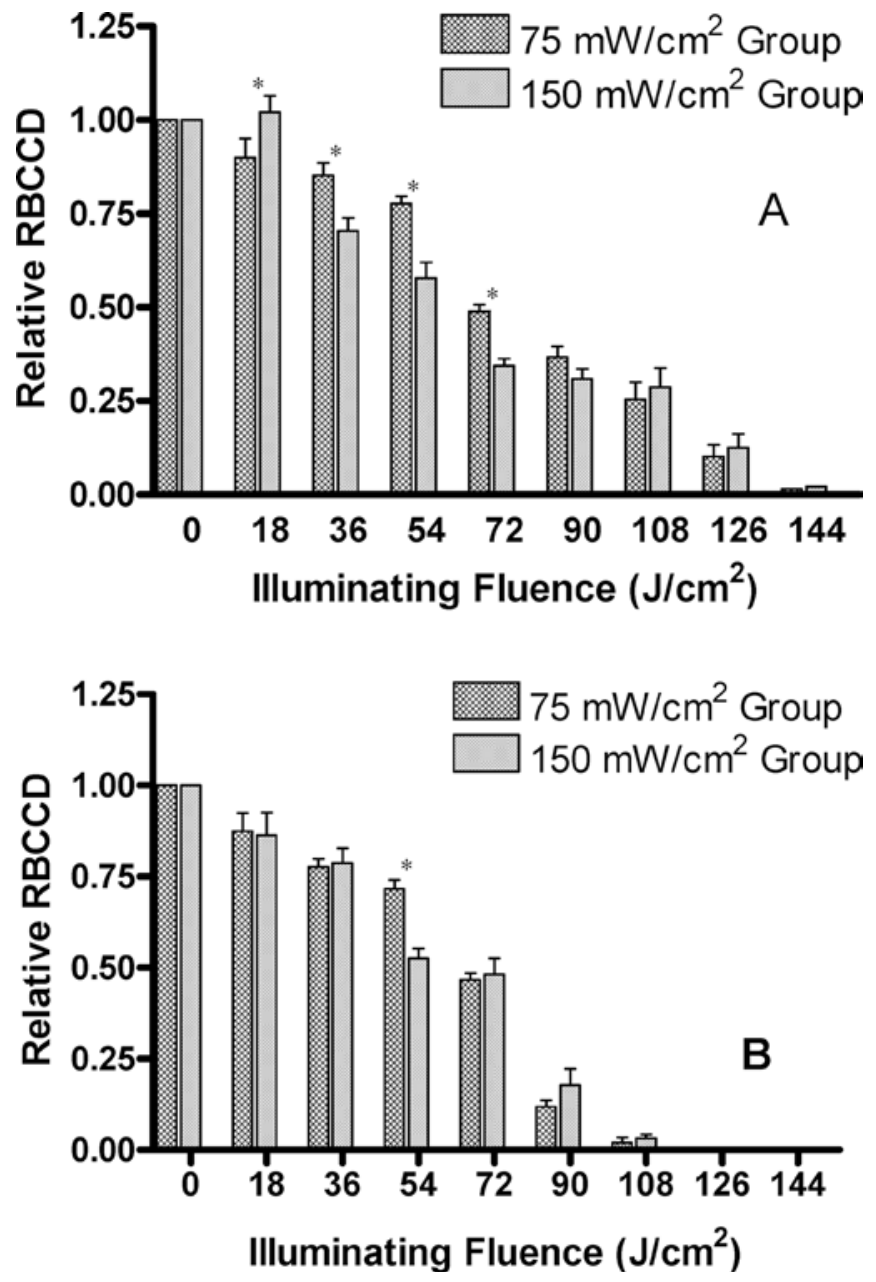

Fig. 1a,b $R B C C D_{\text {rel }}$ changes against light fluence at different fluence rates for arterioles (a) and venules (b). Values are means $\pm \mathrm{SD}$ of ten observation results in each experimental group. Those data that show, based on the Wilcoxon signed rank test, a statistically significant difference between the 75 and $150 \mathrm{~mW} / \mathrm{cm}^{2}$ group are indicated with an asterisk

early period of PDT, while there was no significant difference between the two kinds of illumination near the end of the treatment. The $\mathrm{RBCCD}_{\text {rel }}$ of arterioles was obviously lower under $150 \mathrm{~mW} / \mathrm{cm}^{2}$ illumination than under $75 \mathrm{~mW} / \mathrm{cm}^{2}$ when light doses were $36 \mathrm{~J} / \mathrm{cm}^{2}$ $(p=0.0031), \quad 54 \mathrm{~J} / \mathrm{cm}^{2}(p=0.0024)$, and $72 \mathrm{~J} / \mathrm{cm}^{2}(p=$ $0.036)$. The administration of light doses up to $90 \mathrm{~J} / \mathrm{cm}^{2}$ and above seemed to cause similar damage to arterioles at both light fluence rates. At the light dose of $18 \mathrm{~J} / \mathrm{cm}^{2}$, an increase of $\mathrm{RBCCD}_{\text {rel }}$ occurred under $150 \mathrm{~mW} / \mathrm{cm}^{2}$ illumination, which might be due to a physiological reaction such as the vasospasm-induced subsequent dilation observed during PDT by Wang et al. [15] and the increases in pulse caused by the photosensitizer injection [16]. The $\mathrm{RBCCD}_{\text {rel }}$ of venules decreased more quickly against light dose than that of the arterioles, as shown in Fig. 1b, while no statistically significant difference in $\mathrm{RBCCD}$ rel of venules was observed under 150 and $75 \mathrm{~mW} / \mathrm{cm}^{2}$ illumination, except at light fluence of $54 \mathrm{~J} / \mathrm{cm}^{2}$. It was observed that under the illumination of 
A:

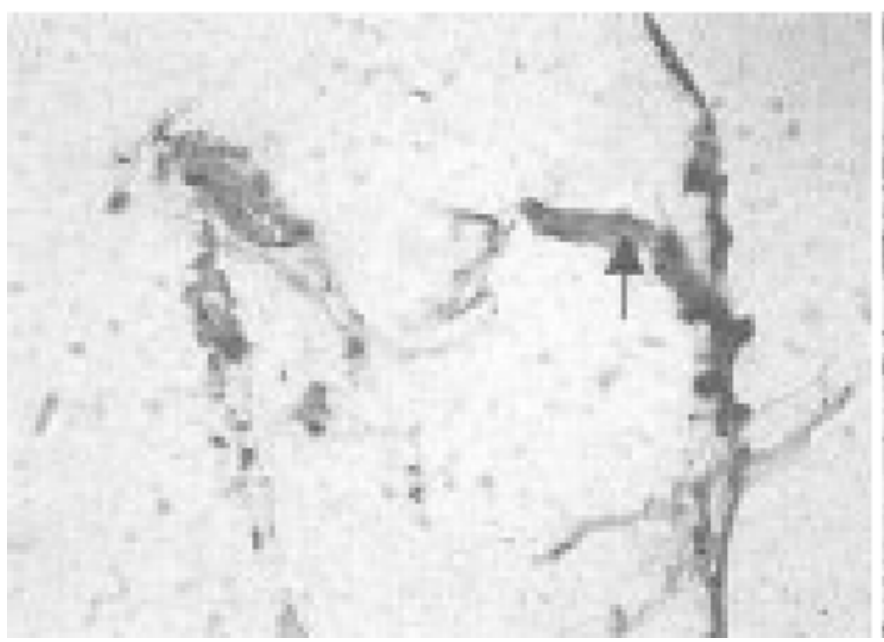

Fig. 2a,b Photomicrographs of vessel damage immediately after PDT induced by $75-\mathrm{mW} / \mathrm{cm}^{2}$ (a) and $150-\mathrm{mW} / \mathrm{cm}^{2}$ (b) illumination. Microvasculature damage of both tumor samples were accompanied with thrombus, as indicated by arrows

$75 \mathrm{~mW} / \mathrm{cm}^{2}$ light, the RBCCD of venules dropped slowly when the illuminating fluence was less than $54 \mathrm{~J} /$ $\mathrm{cm}^{2}$, but rapidly when between 54 and $72 \mathrm{~J} / \mathrm{cm}^{2}$. Therefore, we could assume that at $75 \mathrm{~mW} / \mathrm{cm}^{2}$, the threshold energy dose causing severe damage to the vessels was between 54 and $72 \mathrm{~J} / \mathrm{cm}^{2}$. Similarly, at $150 \mathrm{~mW} / \mathrm{cm}^{2}$, the threshold might be between 36 and $54 \mathrm{~J} / \mathrm{cm}^{2}$.

\section{Vessel histology}

Damage to vessels was evaluated histologically to show the status of microvasculature at the end of PDT at both fluence rates. From all of the six samples from both groups, extensive platelet aggregation and red blood cell agglutination could be observed, and in five of them thrombus occurred. Two typical photos of the samples are shown in Fig. 2. Vessel damage in both groups indicated that microvasculature would eventually be destroyed if the total light fluence was high enough, regardless of the illuminating fluence rates.

Fraction surviving of MDAMB 543 cells, against different fluence rates

To determine the direct MDAMB 543 cell-killing rates induced by PDT at various fluence rates, the survival rates were examined, with the mean OD value being measured as the scale of the fraction surviving. For the control group it was the average OD of the 82 wells in the control plate. OD value of each well in the plates was measured and normalized to produce the mean control value. For the $150 \mathrm{~mW} / \mathrm{cm}^{2}$ illuminated group, the

\section{B:}

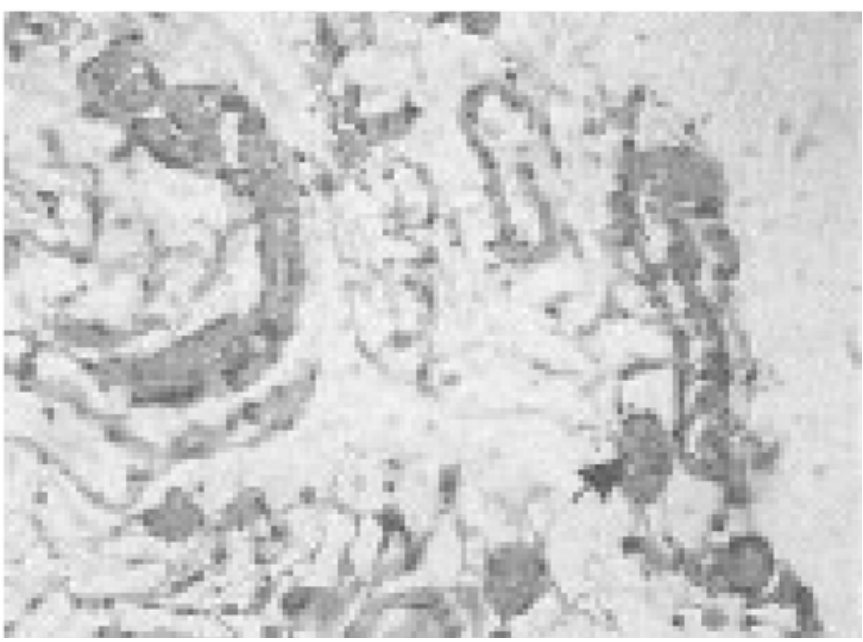

number of wells was 92 and for the $75 \mathrm{~mW} / \mathrm{cm}^{2}$ illuminated group it was 94, where the illuminating light dose was $4.5 \mathrm{~J} / \mathrm{cm}^{2}$. Mean value and standard deviation of the two groups of normalized OD were calculated and are summarized in Fig. 3. It can be seen that the MDAMB 543 cell-survival fraction at a fluence rate of $75 \mathrm{~mW} / \mathrm{cm}^{2}$ is less than that at $150 \mathrm{~mW} / \mathrm{cm}^{2}(p=0.021)$, which indicates a more effective cell-killing under lower fluence rate illumination in PDT.

\section{Discussion}

This work indicates that low fluence rate treatments can be more effective in decreasing vascular lesions than high fluence rate treatments, if the same light fluence is applied during the PDT process. Other studies have also concluded lower fluence rate treatments can preserve the status of oxygen for a more effective PDT [6, 9, 17, 18], and it has been recognized that light fluence rates play a

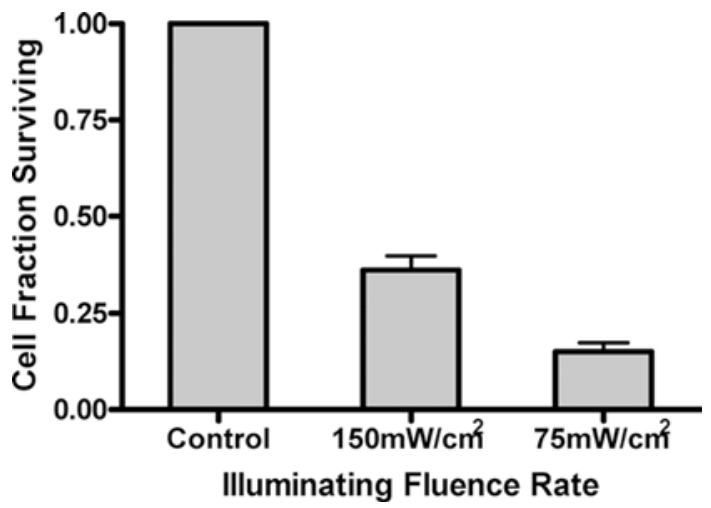

Fig. 3 Surviving cell fraction after illumination with light fluence of $4.5 \mathrm{~J} / \mathrm{cm}^{2}$ in PDT. Values were experimental OD normalized to the control group that was protected from light. Cell fractions surviving in the two experimental groups were significantly different $(p=0.021)$ 
major role in the tumor oxygenation status during PDT exposure. Our work explains the mechanism of oxygen preservation under low fluence illumination in PDT to some extent. It can be concluded from Fig. 1 that lower fluence brings less microvasculature damage during the PDT process, though there seems to be no difference in venules at the two different fluence rates, which may be due to the extremely vulnerable venule circulation. In theory, less damage to microvasculature indicates timely oxygen supply to recover the photochemical consumption of oxygen in PDT. As tissue oxygen is one of the elementary factors of PDT, the timely supply of oxygen is fairly important for the destruction of tumors.

The result of vessel histology studies showed final damage of microvasculature, which occurred in both experimental groups when PDT was completed. This indicates that the light fluence rate has little influence on the final damage to microvasculature if the illumination light dose is sufficient. One would conclude that vascular damage in the tumor focus would be beneficial for longterm tumor control after the completion of PDT. Thus, lower fluence rate illumination can not only reduce the microvasculature damage during PDT to preserve oxygen levels for effective treatment but can induce microvasculature damage to limit the metabolism of tumor cells after PDT. Both of these effects will contribute to tumor destruction. Some clinical and experimental data have been reported recently pointing to a significant tumor cure effect $[12,19]$. From the result of our cellkilling experiment, we can conclude that lower fluence rate treatments may be more effective in increasing the killing rate of tumor cells, which directly indicates better local tumor control. Because the cell-killing experiment was performed in vitro, the oxygen consumption of photosensitizer and the photosensitizer concentration in the medium could be regarded as constant. Thus the cellkilling effect might vary against the excited rate of the photosensitizer molecule only. Characteristics related to light, such as the photobleaching induced by light and the light absorbency of photosensitizer, may be the major influences on the cell-killing effect. The fact that a higher cell-killing rate was obtained under $75 \mathrm{~mW} /$ $\mathrm{cm}^{2}$ illumination, as shown in Fig. 3, indicated less photobleaching or more light absorbency of photosensitizer in lower fluence illuminating PDT, which was consistent with the previous conclusion that the rate of photobleaching increased with decreasing fluence rate at fluence rates of $50-150 \mathrm{~mW} / \mathrm{cm}^{2}$ [19].

Acknowledgements The authors thank Professor Ying $\mathrm{Gu}$ for assistance with the experiments.

\section{References}

1. Thomas JD, Charles JG, Barbara WH, Giulio J, David K, Mladen K, Johan M, Qian P (1998) Photodynamic therapy. J Natl Cancer Inst 90:889-905
2. Rosenthal DI, Glatstein E (1994) Clinical applications of photodynamic therapy. Ann Med 26:405-409

3. Brian WP, Lothar L, Michael SP, Brian CW, Tayyaba H (1997) Absorbed photodynamic dose from pulsed versus continuous wave light examined with tissue-simulating dosimeters. Appl Opt 36(28):7257-7269

4. Curnow A, Haller JC, Bown SG (2000) Oxygen monitoring during 5-aminolaevulinic acid induced photodynamic therapy in normal rat colon comparison of continuous and fractionated light regimes. J Photochem Photobiol B Biol 58:149-155

5. Henderson BW, Busch TM, Vaughan LA, Frawley NP, Babich D, Sosa TA, Zollo JD, Dee AS, Cooper MT, Bellnier DA, Greco WR, Oseroff AR (2000) Photofrin photodynamic therapy can significantly deplete or preserve oxygenation in human basal cell carcinomas during treatment, depending on fluence rate. Cancer Res 60:525-529

6. Foster TH, Murant RS, Bryant RG, Knox RS, Gibson SL, Halif R (1991) Oxygen consumption and diffusion effects in photodynamic therapy. Radiat Res 126:296-303

7. Schunck T, Poulet P (2000) Oxygen consumption through metabolism and photodynamic reactions in cell cultured on microbeads. Phys Med Biol 45:103-119

8. Hendersonx A, Busch T, Oseroff AR (1998) Effects of fluence rate on tumour oxygenation and vascular responses to photodynamic therapy (PDT). In: INABIS ' 98 - 5th Internet world congress on biomedical sciences at McMaster University, Canada, 7-16 December 1998, invited symposium

9. Sitnik TM, Hampton JA, Henderson BW (1998) Reduction of tumour oxygenation during and after photodynamic therapy in vivo: effects of fluence rate. Br J Cancer 77:1386-1394

10. Foster TH, Hartley DF, Nicholas MG, Halif R (1993) Fluence rate effects in photodynamic therapy of multicell tumour spheroids. Cancer Res 53:1249-1254

11. Veenhuizen RB, Stewart FA (1995) The importance of fluence rate photodynamic therapy: was there a parallel with ionising radiation dose rate effects? Radiother Oncol 37:131-135

12. Langmack K, Mehta R, Twyman P, Norris P (2001) Topical photodynamic therapy at low fluence rates - theory and practice. J Photochem Photobiol B Biol 60:37-43

13. Fingar VH, Kik PK, Haydon PS, Cerrito PB, Tseng M, Abang E, Wieman TJ (1999) Analysis of acute vascular damage after photodynamic therapy using benzoporphyrin derivative (BPD). Br J Cancer 79(11-12):1702-1708

14. Fingar VH, Wieman TJ, Wiehle SA, Cerrito PB (1992) The role of microvascular damage in photodynamic therapy: the effect of treatment on vessel constriction, permeability and leukocyte adhesion. Cancer Res 52:4914-4921

15. Wang I, Andersson-Engels S, Nilsson GE, Wardell K, Svanberg K (1997) Superficial blood flow following photodynamic therapy of malignant non-melanoma skin tumours measured by laser Doppler perfusion imaging. Br J Dermatol 136:184 189

16. Webber J, Kessel D, Fromm D (1997) Side effects and photosensitization of human tissues after aminolevulinic acid. J Surg Res 68:31-37

17. Tromberg BJ, Orenstein A, Kimel S, Barker SJ, Hyatt J, Nelson JS, Berns MW (1990) In vivo tumour oxygen tension measurements for the evaluation of the efficiency of photodynamic therapy. Photochem Photobiol 52:375-385

18. Sitnik T, Henderson BW (1998) The effect of fluence rate on tumour and normal tissue responses to photodynamic therapy. Photochem Photobiol 67:462-466

19. Robinson DJ, de Bruijn HS, van der Veen N, Stringer MR, Brown SB, Star WM (1998) Fluorescence photobleaching of ALA-induced protoporphyrin IX during photodynamic therapy of normal hairless mouse skin: the effect of light dose and irradiance and the resulting biological effect. Photochem Photobiol 67:140-149 